\title{
The impact of poverty transitions on frailty among older adults in South Korea: findings from the Korean longitudinal study of ageing
}

Hin Moi Youn ${ }^{1,2}$, Hyeon Ji Lee ${ }^{1,2}$, Doo Woong Lee ${ }^{1,2}$ and Eun-Cheol Park ${ }^{2,3^{*}}$

\begin{abstract}
Background: Frailty is an emerging public health concern among aging populations. Although socioeconomic status is a well-known contributor to frailty, there is limited research investigating the effects of poverty on frailty. This study aimed to examine the association between poverty transitions and frailty prevalence in older adults.

Methods: Data were collected from the six-wave Korean Longitudinal Study of Ageing (2006-2016). A total of 9263 middle-aged and older adults were included in the analysis. Poverty was defined as being below $50 \%$ of the median household income based on the equivalized household. Frailty was measured using an instrument comprising items on physical phenotype (grip strength) and psychological (exhaustion) and social aspects (isolation). Analyses using generalized estimating equations were conducted to estimate the relationship between poverty transition and frailty status.

Results: Among the 9263 respondents, 9.4\% of the male respondents $(n=388)$ and $13.6 \%$ of the female respondents $(n=700)$ were frail. After controlling for covariates, female participants who transitioned into poverty $(\mathrm{OR}=1.31,95 \% \mathrm{Cl}: 1.02-1.69)$ and persistently remained in poverty $(\mathrm{OR}=1.36,95 \% \mathrm{Cl}: 1.10-1.68)$ showed increased odds of frailty in the follow-up year. We did not find significant results in the male participants.

Conclusions: The findings suggest that those who experience poverty transitions, enter poverty, and remain in poverty persistently are at higher risk of frailty. To improve age-related health status among the elderly, interventions aiming to prevent and reduce frailty among the elderly should target individuals who are more vulnerable to the negative effects of frailty.
\end{abstract}

Keywords: Frailty, Frail, Poverty, Elderly, Socioeconomic status, Healthy aging

\footnotetext{
* Correspondence: ecpark@yuhs.ac

${ }^{2}$ Institute of Health Services Research, Yonsei University, 50 Yonsei-ro,

Seodaemun-gu, Seoul 03722, Republic of Korea

${ }^{3}$ Department of Preventive Medicine and Institute of Health Services

Research, Yonsei University College of Medicine, 50 Yonsei-ro,

Seodaemun-gu, Seoul 03722, Republic of Korea

Full list of author information is available at the end of the article
}

C C The Author(s). 2020 Open Access This article is licensed under a Creative Commons Attribution 4.0 International License, which permits use, sharing, adaptation, distribution and reproduction in any medium or format, as long as you give appropriate credit to the original author(s) and the source, provide a link to the Creative Commons licence, and indicate if changes were made. The images or other third party material in this article are included in the article's Creative Commons licence, unless indicated otherwise in a credit line to the material. If material is not included in the article's Creative Commons licence and your intended use is not permitted by statutory regulation or exceeds the permitted use, you will need to obtain permission directly from the copyright holder. To view a copy of this licence, visit http://creativecommons.org/licenses/by/4.0/. The Creative Commons Public Domain Dedication waiver (http://creativecommons.org/publicdomain/zero/1.0/) applies to the data made available in this article, unless otherwise stated in a credit line to the data. 


\section{Background}

Population aging is a global phenomenon. Increased life expectancy and low birth rates have contributed to the world's aging population, and virtually every country is experiencing growth in the proportion of older people in their population [1]. South Korea has one of the fastest aging populations - the proportion of people aged 65 or older is projected to reach $46.5 \%$ by 2067 , rendering it the world's most aged country [2]. Although increased life expectancy is a major achievement, it presents challenges regarding the burden of age-related diseases, such as health costs and social care. The prevention and treatment of frailty has recently received increasing attention with respect to the promotion of healthier aging among the elderly [3]. Frailty develops as a consequence of agerelated decline in multiple physiological systems, which collectively results in vulnerability to adverse health status changes triggered by stressor events [4]. Frail individuals are at a greater risk of adverse health outcomes such as falls, incident disability, institutionalization, hospitalization, and death, and have a greater need for healthcare [5]. Thus, frailty can have consequences for quality of life, health, and welfare systems.

The incidence of frailty may vary diversely among individuals in that some are more vulnerable to stressors whereas others are resilient. Frailty in the elderly is a multidimensional syndrome that involves the interaction of biological, psychological, and social factors [4]. To date, various studies have shown that socioeconomic status (including education, occupation, and income) is an important contributor to the disparities in frailty among the elderly [5-9]. Although it is well recognized that poverty has important implications for health, few studies have focused on poverty and its effect on frailty [10]. Moreover, there has been limited research on the effect of changes in poverty status over time. Because frailty development is influenced by the aging-related accumulation of deficits, longitudinal assessment can provide a more robust understanding of the extent to which poverty is associated with frailty.

While a higher prevalence of elderly poverty is a global phenomenon, South Korea has the highest poverty rate of people aged over 65 among the Organization for Economic Co-operation and Development (OECD) countries. In 2017, the country's elderly poverty rate, which indicates the proportion of senior citizens earning below $50 \%$ of the overall median income, reached $43.8 \%$, whereas the average poverty rate in the OECD was $13.5 \%$ [11]. Therefore, better understanding of the effects of poverty on frailty is necessary for the development of intervention strategies aimed at preventing and reducing frailty and its burden on individuals, especially those in poverty. In this study, we sought to assess the prevalence of frailty in middle-aged and older adults and examine the impact of poverty transition on frailty.

\section{Methods}

\section{Data source and study population}

Data were collected from the Korean Longitudinal Study of Ageing (KLoSA), which was conducted in 2006, 2008, 2010, 2012, 2014, and 2016. The KLoSA is a large-scale, longitudinal survey of the population aged 45 and older living in households selected by multistage stratified probability sampling to ensure national representativeness. It was designed to help develop policies to address health and social issues that emerged because of rapid population aging. In the 2006 baseline survey, the original sample of 10,254 respondents completed interviews by well-trained interviewers. The household response rate was $70.7 \%$ and the individual response rate within households was $75.4 \%$. This survey was followed up with 8875,8229 , and 7813 respondents in 2008, 2010, and 2012, respectively. A refreshment sample of 920 individuals born in 1962 or 1963 was introduced in 2014 and was included in the 2014 and 2016 waves. The combined sample included 8387 respondents in 2014 and 7893 in 2016 [12]. After excluding those with missing data and those who were unable to follow up, 9263 respondents were included in the present sample.

\section{Measures \\ Poverty transitions}

The variable of interest was the transition of poverty status across time. We employed a relative measure of poverty, defining it as earning below $50 \%$ of the median household income based on the equivalized household. The value of the poverty line was set for each year (2006, 2008, 2010, 2012, 2014, 2016) based on data from Statistics Korea. The KLoSA contains detailed information about the different types of income that comprise aggregate income, including earned income, asset income, public transfer income, financial support, and other types of income. Total household income is the sum of the incomes of all household members living together, including the respondent. The household income reported by the representative member was assigned to all the other members such that that the total amount of household income had the same value across all household members [12]. In the present study, we used equivalized household income, which considers the square root of the number of household members. The current equivalized household income of all respondents in the sample was allocated into poverty and non-poverty groups based on the previously defined poverty line. Poverty transition was measured as change in poverty status in a previous year $(\mathrm{Y}-1)$ and the subsequent year (Y0). We categorized the respondents into four groups: non-poverty to non-poverty (NN, persistence of nonpoverty), poverty to non-poverty ( $\mathrm{PN}$, exiting poverty), 
non-poverty to poverty (NP, transition to poverty), and poverty to poverty (PP, persistence of poverty) [13].

\section{Frailty}

We used a broader definition of frailty that includes physical phenotype and social and psychological aspects. The frailty instrument consists of items measuring weakness of grip strength, exhaustion, and social isolation. It was developed to assess the risks of adverse health outcomes such as disability, institutionalization, and mortality of older adults with high predictive validity, discrimination, and calibration ability. The validity of the frailty instrument has been reported elsewhere [14]. Weakness was measured using grip strength $(<24 \mathrm{~kg}$ for men and $<15 \mathrm{~kg}$ for women). Exhaustion was evaluated by self-reporting either the feeling that every task required effort or that they could not "get going" in the preceding week. Isolation was assessed by asking about participation in meetings or group activities. The scale scores ranged from 0 to 3 and were categorized as frail $(\geq 2)$, pre-frail $(\geq 1)$, and robust (0) [15]. In this study, we grouped participants into two categories: frail $(\geq 2)$ and non-frail $(\leq 1)$.

\section{Covariates}

Demographic, socioeconomic, and health-related factors were included in the study. The demographic variables were sex, age $(45-64,65-74,75$ or older), marital status (with spouse, without spouse), and number of household members $(1,2,3$, or more). The socioeconomic variables included educational level (elementary school or below, middle/high school, college, or above), household income (quantiles), current economic activity (active, inactive), region (metropolitan, urban, rural), and health insurance (national health insurance, medical aid). The healthrelated factors included smoking (yes, no), drinking (yes, no), perceived health status (healthy, average, unhealthy), and presence of chronic diseases (yes, no). Chronic diseases included hypertension, diabetes, malignant tumor, liver disease, cardiovascular disease, cerebrovascular disease, psychiatric disorders, and rheumatoid arthritis. We used indicators of individuals' functional and cognitive status, including activities of daily living [independent, needs help/difficulty with activities of daily living (ADL)], instrumental activities of daily living [independent, needs help/difficulty with instrumental ADL (IADL)], and cognitive impairment (yes, no). Cognitive impairment was measured using the Korean Mini-Mental State Examination (K-MMSE), which includes 11 items in seven categories of cognitive functions (orientation of time and place, registration, attention and calculation, recall, language, and visual construction). The total score range from 0 to 30 , and higher scores indicate better cognitive function. The validity of the K-MMSE has been reported elsewhere [16]. We followed the conventional classification criteria and categorized scores as indicating normal cognitive function (K-MMSE $\geq 24$ ) and mild to severe cognitive impairment (K-MMSE $\leq 23)$. Frailty status in the previous year was included to take account of its contribution to frailty in the follow-up year.

\section{Statistical analysis}

The distribution of general characteristics was calculated at baseline. Differences in baseline characteristics between non-frail and frail respondents were determined using $X^{2}$ tests. To evaluate repeatedly measured individuals, PROC GENMOD was used to employ a generalized estimating equation (GEE) for repeated measure analysis. We evaluated whether the probability of frailty changed after poverty transitions over two consecutive years (between 2006 and 2008, 2008-2010, 2010-2012, 20122014, or 2014-2016). Furthermore, subgroup analyses stratified by age, marital status, current economic activity, region, presence of chronic diseases, and cognitive impairment were performed to examine the association between poverty transitions and frailty after adjusting for covariates. All analyses were conducted using SAS software, version 9.3 (SAS Institute, Cary, NC).

\section{Results}

Table 1 presents the baseline characteristics of the study population. Among the total 9263 participants, 44.4\% (4115) were men and 55.6\% (5148) were women. Overall, $388(9.4 \%)$ men and $700(13.6 \%)$ women were frail. With regard to poverty status, 2111 (51.3\%) men and 2894 (56.2\%) women were below the poverty threshold. Among those in poverty, women showed a greater proportion of frailty $(18.8 \%)$ than men (15.1\%). Across age groups, the oldest group had the highest proportion of frailty in both men (29.8\%) and women (36.6\%). Furthermore, the lower income quantiles showed smaller proportions of frail individuals among both men and women (15.6 and 20.3\% for men and women in Quantile 1 , respectively). Table 2 shows the results of the GEE model for the impact of poverty transitions on frailty. Among women, those who were not in poverty in the previous year but entered poverty in the subsequent year $(\mathrm{PN})(\mathrm{OR}=1.31,95 \% \mathrm{CI}: 1.02-1.69)$ and those who were persistently in poverty $(\mathrm{PP})(\mathrm{OR}=1.36,95 \% \mathrm{CI}$ : 1.10 1.68) showed increased odds of frailty compared with those who were persistently not in poverty (NN). Among men, there was no statistically significant relationship between poverty transitions and frailty. However, although not statistically significant, those who were persistently in poverty showed the highest odds of frailty (OR $=1.23$, 95\% CI: 0.94-1.62). Men aged 75 years or older had the highest odds of frailty $(\mathrm{OR}=2.40,95 \% \mathrm{CI}$ : 1.87-3.09) while women aged between 65 and 74 had the highest odds of frailty $(\mathrm{OR}=1.24,95 \% \mathrm{CI}$ : $1.06-$ 
Table 1 General characteristics of the study population (2006 baseline)

\begin{tabular}{|c|c|c|c|c|c|c|c|c|c|c|c|c|c|c|}
\hline \multirow[t]{5}{*}{ Variables } & \multicolumn{14}{|c|}{ Frailty $^{a}$} \\
\hline & \multicolumn{6}{|l|}{ Male } & \multirow{4}{*}{$\begin{array}{l}p \\
\text { value }\end{array}$} & \multicolumn{6}{|c|}{ Female } & \multirow{4}{*}{$\begin{array}{l}p \\
\text { value }\end{array}$} \\
\hline & \multicolumn{2}{|l|}{ Total } & \multicolumn{2}{|l|}{ Yes } & \multicolumn{2}{|l|}{ No } & & \multicolumn{2}{|l|}{ Total } & \multicolumn{2}{|l|}{ Yes } & \multicolumn{2}{|l|}{ No } & \\
\hline & $\mathrm{N}$ & $\%$ & $\mathrm{~N}$ & $\%$ & $\mathrm{~N}$ & $\%$ & & $\mathrm{~N}$ & $\%$ & $\mathrm{~N}$ & $\%$ & $\mathrm{~N}$ & $\%$ & \\
\hline & 4115 & 100.0 & 388 & 9.4 & 3727 & 90.6 & & 5148 & 100.0 & 700 & 13.6 & 4448 & 86.4 & \\
\hline Current relative poverty $^{b}$ & & & & & & & $<.0001$ & & & & & & & $<.0001$ \\
\hline Non-poverty & 2004 & 48.7 & 69 & 3.4 & 1935 & 96.6 & & 2254 & 43.8 & 155 & 6.9 & 2099 & 93.1 & \\
\hline Poverty & 2111 & 51.3 & 319 & 15.1 & 1792 & 84.9 & & 2894 & 56.2 & 545 & 18.8 & 2349 & 81.2 & \\
\hline Age & & & & & & & $<.0001$ & & & & & & & $<.0001$ \\
\hline $45-64$ & 2554 & 62.1 & 104 & 4.1 & 2450 & 95.9 & & 3177 & 61.7 & 167 & 5.3 & 3010 & 94.7 & \\
\hline $65-74$ & 1125 & 27.3 & 154 & 13.7 & 971 & 86.3 & & 1285 & 25.0 & 282 & 21.9 & 1003 & 78.1 & \\
\hline$\geq 75$ & 436 & 10.6 & 130 & 29.8 & 306 & 70.2 & & 686 & 13.3 & 251 & 36.6 & 435 & 63.4 & \\
\hline Marital status & & & & & & & $<.0001$ & & & & & & & $<.0001$ \\
\hline With spouse & 3788 & 92.1 & 307 & 8.1 & 3481 & 91.9 & & 3571 & 69.4 & 299 & 8.4 & 3272 & 91.6 & \\
\hline Without spouse & 327 & 7.9 & 81 & 24.8 & 246 & 75.2 & & 1577 & 30.6 & 401 & 25.4 & 1176 & 74.6 & \\
\hline No. of household members & & & & & & & $<.0001$ & & & & & & & $<.0001$ \\
\hline 1 & 139 & 3.4 & 36 & 25.9 & 103 & 74.1 & & 632 & 12.3 & 156 & 24.7 & 476 & 75.3 & \\
\hline 2 & 1706 & 41.5 & 203 & 11.9 & 1503 & 88.1 & & 1820 & 35.4 & 240 & 13.2 & 1580 & 86.8 & \\
\hline$\geq 3$ & 2270 & 55.2 & 149 & 6.6 & 2121 & 93.4 & & 2696 & 52.4 & 304 & 11.3 & 2392 & 88.7 & \\
\hline Educational level & & & & & & & $<.0001$ & & & & & & & $<.0001$ \\
\hline Elementary school graduate or lower & 1260 & 30.6 & 252 & 20.0 & 1008 & 80.0 & & 2886 & 56.1 & 619 & 21.4 & 2267 & 78.6 & \\
\hline Middle/high school graduate & 2125 & 51.6 & 110 & 5.2 & 2015 & 94.8 & & 1998 & 38.8 & 77 & 3.9 & 1921 & 96.1 & \\
\hline College graduate or higher & 730 & 17.7 & 26 & 3.6 & 704 & 96.4 & & 264 & 5.1 & 4 & 1.5 & 260 & 98.5 & \\
\hline Current economic activity & & & & & & & $<.0001$ & & & & & & & $<.0001$ \\
\hline Active & 2441 & 59.3 & 84 & 3.4 & 2357 & 96.6 & & 1298 & 25.2 & 90 & 6.9 & 1208 & 93.1 & \\
\hline Inactive & 1674 & 40.7 & 304 & 18.2 & 1370 & 81.8 & & 3850 & 74.8 & 610 & 15.8 & 3240 & 84.2 & \\
\hline Region & & & & & & & $<.0001$ & & & & & & & 0.0014 \\
\hline Metropolitan & 1800 & 43.7 & 152 & 8.4 & 1648 & 91.6 & & 2353 & 45.7 & 310 & 13.2 & 2043 & 86.8 & \\
\hline Urban & 1390 & 33.8 & 113 & 8.1 & 1277 & 91.9 & & 1673 & 32.5 & 202 & 12.1 & 1471 & 87.9 & \\
\hline Rural & 925 & 22.5 & 123 & 13.3 & 802 & 86.7 & & 1122 & 21.8 & 188 & 16.8 & 934 & 83.2 & \\
\hline Health insurance type & & & & & & & $<.0001$ & & & & & & & $<.0001$ \\
\hline $\mathrm{NH} \mathrm{I}^{\mathrm{c}}$ & 3907 & 94.9 & 323 & 8.3 & 3584 & 91.7 & & 4838 & 94.0 & 592 & 12.2 & 4246 & 87.8 & \\
\hline Medical aid & 208 & 5.1 & 65 & 31.3 & 143 & 68.8 & & 310 & 6.0 & 108 & 34.8 & 202 & 65.2 & \\
\hline Household income $^{d}$ & & & & & & & $<.0001$ & & & & & & & $<.0001$ \\
\hline Quantile 1 (low) & 1695 & 41.2 & 265 & 15.6 & 1430 & 84.4 & & 2393 & 46.5 & 485 & 20.3 & 1908 & 79.7 & \\
\hline Quantile 2 & 902 & 21.9 & 80 & 8.9 & 822 & 91.1 & & 1078 & 20.9 & 121 & 11.2 & 957 & 88.8 & \\
\hline Quantile 3 & 551 & 13.4 & 19 & 3.4 & 532 & 96.6 & & 599 & 11.6 & 39 & 6.5 & 560 & 93.5 & \\
\hline Quantile 4 & 439 & 10.7 & 13 & 3.0 & 426 & 97.0 & & 504 & 9.8 & 31 & 6.2 & 473 & 93.8 & \\
\hline Quantile 5 (high) & 528 & 12.8 & 11 & 2.1 & 517 & 97.9 & & 574 & 11.1 & 24 & 4.2 & 550 & 95.8 & \\
\hline Smoking & & & & & & & 0.6453 & & & & & & & $<.0001$ \\
\hline No & 1593 & 38.7 & 146 & 9.2 & 1447 & 90.8 & & 4956 & 96.3 & 642 & 13.0 & 4314 & 87.0 & \\
\hline Yes & 2522 & 61.3 & 242 & 9.6 & 2280 & 90.4 & & 192 & 3.7 & 58 & 30.2 & 134 & 69.8 & \\
\hline Drinking & & & & & & & 0.2594 & & & & & & & 0.0445 \\
\hline No & 986 & 24.0 & 102 & 10.3 & 884 & 89.7 & & 4035 & 78.4 & 569 & 14.1 & 3466 & 85.9 & \\
\hline Yes & 3129 & 76.0 & 286 & 9.1 & 843 & 90.9 & & 1113 & 21.6 & 131 & 11.8 & 982 & 88.2 & \\
\hline
\end{tabular}


Table 1 General characteristics of the study population (2006 baseline) (Continued)

\begin{tabular}{|c|c|c|c|c|c|c|c|c|c|c|c|c|c|c|}
\hline \multirow[t]{4}{*}{ Variables } & \multicolumn{14}{|c|}{ Frailty $^{a}$} \\
\hline & \multicolumn{6}{|l|}{ Male } & \multirow{4}{*}{$\begin{array}{l}\boldsymbol{p} \\
\text { value }\end{array}$} & \multicolumn{6}{|c|}{ Female } & \multirow{4}{*}{$\begin{array}{l}p \\
\text { value }\end{array}$} \\
\hline & \multicolumn{2}{|l|}{ Total } & \multicolumn{2}{|l|}{ Yes } & \multicolumn{2}{|l|}{ No } & & \multicolumn{2}{|l|}{ Total } & \multicolumn{2}{|l|}{ Yes } & \multicolumn{2}{|l|}{ No } & \\
\hline & $\mathrm{N}$ & $\%$ & $\mathrm{~N}$ & $\%$ & $\mathrm{~N}$ & $\%$ & & $\mathrm{~N}$ & $\%$ & $\mathrm{~N}$ & $\%$ & $\mathrm{~N}$ & $\%$ & \\
\hline & 4115 & 100.0 & 388 & 9.4 & 3727 & 90.6 & & 5148 & 100.0 & 700 & 13.6 & 4448 & 86.4 & \\
\hline \multicolumn{7}{|l|}{ Perceived health status } & $<.0001$ & & & & & & & $<.0001$ \\
\hline Healthy & 680 & 16.5 & 14 & 2.1 & 666 & 97.9 & & 565 & 11.0 & 12 & 2.1 & 553 & 97.9 & \\
\hline Average & 1685 & 40.9 & 48 & 2.8 & 1637 & 97.2 & & 1669 & 32.4 & 86 & 5.2 & 1583 & 94.8 & \\
\hline Unhealthy & 1750 & 42.5 & 326 & 18.6 & 1424 & 81.4 & & 2914 & 56.6 & 602 & 20.7 & 2312 & 79.3 & \\
\hline \multicolumn{7}{|l|}{ Chronic disease $^{\mathrm{e}}$} & $<.0001$ & & & & & & & $<.0001$ \\
\hline No & 2349 & 57.1 & 139 & 5.9 & 2210 & 94.1 & & 2598 & 50.5 & 213 & 8.2 & 2385 & 91.8 & \\
\hline Yes & 1766 & 42.9 & 249 & 14.1 & 1517 & 85.9 & & 2550 & 49.5 & 487 & 19.1 & 2063 & 80.9 & \\
\hline \multicolumn{7}{|l|}{ Disability } & $<.0001$ & & & & & & & $<.0001$ \\
\hline No & 3801 & 92.4 & 314 & 8.3 & 3487 & 91.7 & & 4967 & 96.5 & 653 & 13.1 & 4,14 & 86.9 & \\
\hline Yes & 314 & 7.6 & 74 & 23.6 & 240 & 76.4 & & 181 & 3.5 & 47 & 26.0 & 134 & 74.0 & \\
\hline \multicolumn{7}{|l|}{$A D L^{f}$} & $<.0001$ & & & & & & & $<.0001$ \\
\hline Independent & 4022 & 97.7 & 339 & 8.4 & 3683 & 91.6 & & 5011 & 97.3 & 635 & 12.7 & 4376 & 87.3 & \\
\hline Needs help/difficulty with ADL & 93 & 2.3 & 49 & 52.7 & 44 & 47.3 & & 137 & 2.7 & 65 & 47.4 & 72 & 52.6 & \\
\hline \multicolumn{7}{|l|}{$\operatorname{IADL}^{\mathrm{g}}$} & $<.0001$ & & & & & & & $<.0001$ \\
\hline Independent & 3546 & 86.2 & 269 & 7.6 & 3277 & 92.4 & & 4673 & 90.8 & 524 & 11.2 & 4149 & 88.8 & \\
\hline Needs help/difficulty with IADL & 569 & 13.8 & 119 & 20.9 & 450 & 79.1 & & 475 & 9.2 & 176 & 37.1 & 299 & 62.9 & \\
\hline \multicolumn{7}{|l|}{ Cognitive impairment ${ }^{\mathrm{h}}$} & $<.0001$ & & & & & & & $<.0001$ \\
\hline No & 3572 & 86.8 & 226 & 6.3 & 3346 & 93.7 & & 3715 & 72.2 & 277 & 7.5 & 3438 & 92.5 & \\
\hline Yes & 543 & 13.2 & 162 & 29.8 & 381 & 70.2 & & 1433 & 27.8 & 423 & 29.5 & 1010 & 70.5 & \\
\hline \multicolumn{15}{|c|}{$\begin{array}{l}\text { a The frailty instrument consists of grip strength, exhaustion, and social isolation (frail } \geq 2 \text {, non-frail } \leq 1 \text { ) } \\
\text { b Relative poverty line: } 50 \% \text { of median household income based on the equivalized household income } \\
\text { c NHI: National Health Insurance (employee and self-employee insured) } \\
\text { d Participants' current equivalized household income level was allocated into quantile groups based on the data from Statistics Korea } \\
\text { e Chronic diseases include hypertension, diabetes, malignant tumor, liver disease, cardiovascular disease, cerebrovascular disease, psychiatric disorders, and } \\
\text { rheumatoid arthritis disease } \\
\text { f ADL: Activities of daily living } \\
\text { g IADL: Instrumental activities of daily living } \\
\text { h K-MMSE (Korean Mini-Mental State Examination): normal cognitive function (K-MMSE }>24 \text { ) and mild to severe cognitive impairment (K-MMSE }<23 \text { ) }\end{array}$} \\
\hline
\end{tabular}

1.46). Significant relationships were found between household income and frailty. Those in the highest quantile showed the lowest odds of frailty among both men $(\mathrm{OR}=0.41,95 \% \mathrm{CI}: 0.27-0.62)$ and women $(\mathrm{OR}=$ $0.71,95 \%$ CI: 0.52-0.97). Frailty in the previous year was significantly associated with frailty in the subsequent year for both men $(\mathrm{OR}=3.61,95 \% \mathrm{CI}$ : 2.96-4.40) and women $(\mathrm{OR}=3.41,95 \% \mathrm{CI}: 2.98-3.89)$. Figure 1 presents the results for the subgroup analysis of the association between poverty transitions and frailty stratified by region. The results show that, compared to individuals living in urban area, those living in rural and metropolitan areas have greater odds of being frailty. In addition, women show a graded association between poverty transitions and frailty, where persistently remained in poverty had the highest odds in metropolitan $(\mathrm{OR}=1.70$,
95\% CI: 1.19-2.43) and rural (OR = 1.60, 95\% CI: $1.07-$ 2.38).

\section{Discussion}

In this study, we examined whether transitions in poverty status are associated with frailty in middle-aged and older adults in South Korea. As aforementioned, about a half of the Korean elderly population is living in poverty. In our study, we found that more than half of the individuals aged over 45 years were below the poverty threshold. Poverty is a well-known socioeconomic determinant that is intertwined with health. We attempted to shed light on how poverty corresponds to frailty, that is, the adverse health outcomes of accumulated risk factors over the course of a lifetime. The prevalence of frailty in Korea has been reported to range from 2.5 to $31.7 \%$ 
Table 2 Association between poverty transitions and frailty: the results of GEE analysis

\begin{tabular}{|c|c|c|c|c|c|c|c|c|}
\hline \multirow[t]{3}{*}{ Variables } & \multicolumn{8}{|c|}{ Frailty $^{a}$} \\
\hline & \multicolumn{4}{|c|}{ Male } & \multicolumn{4}{|c|}{ Female } \\
\hline & $\overline{O R^{i}}$ & \multicolumn{3}{|c|}{$95 \% \mathrm{Cl}^{\mathrm{i}}$} & $\overline{O^{i}}$ & \multicolumn{3}{|c|}{$95 \% \mathrm{Cl}^{\mathrm{i}}$} \\
\hline \multicolumn{9}{|l|}{ Poverty transition ${ }^{\mathrm{b}}$} \\
\hline Persistence of non-poverty (NN) & 1.00 & & & & 1.00 & & & \\
\hline Exiting poverty (PN) & 1.22 & $(0.93$ & - & 1.59) & 1.16 & $(0.96$ & - & 1.41) \\
\hline Transition to poverty (NP) & 1.03 & $(0.74$ & - & 1.44) & 1.31 & $(1.02$ & - & 1.69) \\
\hline Persistence of poverty (PP) & 1.23 & $(0.94$ & - & 1.62) & 1.36 & $(1.10$ & - & 1.68) \\
\hline
\end{tabular}

\section{Age}

$$
\begin{array}{r}
45-64 \\
65-74
\end{array}
$$

$\geq 75$

\section{Marital status}

With spouse

Without spouse

\section{No. of household members}

1

2

$\geq 3$

Educational level

Elementary school graduate or lower

Middle/high school graduate

College graduate or higher

\section{Current economic activity}

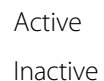

Region

Metropolitan

Urban

Rural

\section{Health insurance type}

$$
\begin{aligned}
& \mathrm{NHI}^{\mathrm{c}} \\
& \text { Medical aid }
\end{aligned}
$$

Household income

Quantile 1 (low)

Quantile 2

Quantile 3

Quantile 4

Quantile 5 (high)

\section{Smoking}

No

Yes

Drinking

No

Yes
1.00

1.40

2.40

(1.12

(1.87

1.00

1.62

(1.28

1.00

0.93

0.98

(0.71

(0.74

1.00

0.83

0.65

$(0.70$

(0.48

1.00

1.40

(1.17

1.00

1.18

1.00

(0.98

(0.82

1.00

1.35

(1.02

1.78)

0.99)

0.88)

1.23)

1.31)

1.76)

1.00

1.24

1.81

(1.06

(1.50

1.46)

3.09)

2.05)

1.00

1.29

$(1.12$

1.49)

1.00

1.12

(0.95

1.13

(0.96

1.00

0.70

(0.60

0.58

(0.33

1.00

1.11

(0.97

1.28)

1.00

1.20

(1.05

1.03

(0.90

1.18)

1.21)

1.00

1.49

(1.22

1.00

0.80

(0.66

1.00

0.58

(0.41

0.96)

0.76

0.82)

0.85

0.92)

0.92

0.62 )

0.71

(0.66

0.63

$(0.27$

0.41

(0.27

1.00

1.24

(1.05

1.46)

1.00

1.54

(1.21

1.00

0.96

(0.80

1.16)

1.00

1.03

(0.90 
Table 2 Association between poverty transitions and frailty: the results of GEE analysis (Continued)

\begin{tabular}{|c|c|c|c|c|c|c|c|c|}
\hline \multirow[t]{3}{*}{ Variables } & \multicolumn{8}{|c|}{ Frailty $^{\mathbf{a}}$} \\
\hline & \multicolumn{4}{|c|}{ Male } & \multicolumn{4}{|c|}{ Female } \\
\hline & $\overline{O R^{i}}$ & \multicolumn{3}{|c|}{$95 \% \mathrm{Cl}^{\mathrm{i}}$} & $\overline{O R^{i}}$ & \multicolumn{3}{|c|}{$95 \% \mathrm{Cl}^{\mathrm{i}}$} \\
\hline \multicolumn{9}{|l|}{ Perceived health status } \\
\hline Healthy & 1.00 & & & & 1.00 & & & \\
\hline Average & 0.99 & $(0.68$ & - & 1.45) & 0.91 & $(0.62$ & - & 1.35) \\
\hline Unhealthy & 2.16 & $(1.47$ & - & 3.16) & 2.78 & $(1.91$ & - & 4.06) \\
\hline \multicolumn{9}{|l|}{ Chronic disease $\mathrm{e}^{\mathrm{e}}$} \\
\hline No & 1.00 & & & & 1.00 & & & \\
\hline Yes & 1.12 & $(0.95$ & - & 1.32) & 1.11 & $(0.97$ & - & 1.27) \\
\hline \multicolumn{9}{|l|}{ Disability } \\
\hline No & 1.00 & & & & 1.00 & & & \\
\hline Yes & 0.94 & $(0.46$ & - & 1.94) & 0.84 & $(0.48$ & - & 1.47) \\
\hline \multicolumn{9}{|l|}{$A D L^{f}$} \\
\hline Independent & 1.00 & & & & 1.00 & & & \\
\hline Needs help/difficulty with ADL & 1.92 & $(1.18$ & - & 3.12) & 1.79 & $(1.29$ & - & 2.49) \\
\hline \multicolumn{9}{|l|}{$I_{A D L^{g}}$} \\
\hline Independent & 1.00 & & & & 1.00 & & & \\
\hline Needs help/difficulty with IADL & 1.30 & $(1.06$ & - & 1.59) & 1.97 & $(1.64$ & - & 2.37) \\
\hline \multicolumn{9}{|l|}{ Cognitive impairment } \\
\hline No & 1.00 & & & & 1.00 & & & \\
\hline Yes & 2.82 & $(2.40$ & - & 3.31) & 2.32 & $(2.06$ & - & 2.62) \\
\hline \multicolumn{9}{|l|}{ Frailty in the previous year } \\
\hline No & 1.00 & & & & 1.00 & & & \\
\hline Yes & 3.61 & (2.96 & - & 4.40) & 3.41 & $(2.98$ & - & 3.89) \\
\hline
\end{tabular}

a The frailty instrument consists of grip strength, exhaustion, and social isolation (frail $\geq 2$, non-frail $\leq 1$ )

${ }^{b}$ Relative poverty line: $50 \%$ of median household income based on the equivalized household income

${ }^{c} \mathrm{NHI}$ National Health Insurance (employee and self-employee insured)

' Participants' current equivalized household income level was allocated into quantile groups based on the data from Statistics Korea

${ }^{\text {e } C h r o n i c ~ d i s e a s e s ~ i n c l u d e ~ h y p e r t e n s i o n, ~ d i a b e t e s, ~ m a l i g n a n t ~ t u m o r, ~ l i v e r ~ d i s e a s e, ~ c a r d i o v a s c u l a r ~ d i s e a s e, ~ c e r e b r o v a s c u l a r ~ d i s e a s e, ~ p s y c h i a t r i c ~ d i s o r d e r s, ~ a n d ~}$

rheumatoid arthritis disease

${ }^{f} A D L$ Activities of daily living

${ }^{9}$ IADL Instrumental activities of daily living

${ }^{h}$ K-MMSE (Korean Mini-Mental State Examination) normal cognitive function (K-MMSE $\geq 24$ ) and mild to severe cognitive impairment (K-MMSE $\leq 23$ )

'OR: odds ratio; $95 \% \mathrm{Cl}$ : confidence interval

depending on the study population and components of each frailty scale $[15,17,18]$. In our study, we used a frailty instrument that measures physical, psychological, and social domains, which can contribute to frailty independently or interactively [19]. The prevalence of frailty was $11.7 \%$ (men $9.4 \%$ and women $13.6 \%$ ). The results indicated that experiencing poverty significantly increased the probability of frailty compared to persistently remaining in non-poverty. The findings are in line with those of previous studies that found that poverty is associated with frailty or poorer physical, psychological, and cognitive functioning $[10,20,21]$ However, significant relationships between poverty transitions and frailty were observed only in women. In general, women are more likely to be at risk related to overall health because of several factors, including age-related diseases, low socioeconomic status, and low activity level. Our results are in accordance with those of previous studies that found women are frailer than men [5, 17, 22, 23]. The finding that poverty affects women and men unequally offers a direction for targeted interventions to prevent or manage symptoms of frailty. It has been shown that the probability of frailty increases with persistent poverty over time [20, 24]. Poverty leads to various health risks such as less knowledge about healthy behaviors, lower access to health services, and environmental risks for illness and disability [25]. Given that frailty develops because of accumulated deficits over time, sustained exposure to risks due to poverty will increase the prevalence of frailty. Furthermore, those who transitioned into poverty in the follow-up year showed an increased probability of frailty as well. A study found that income 


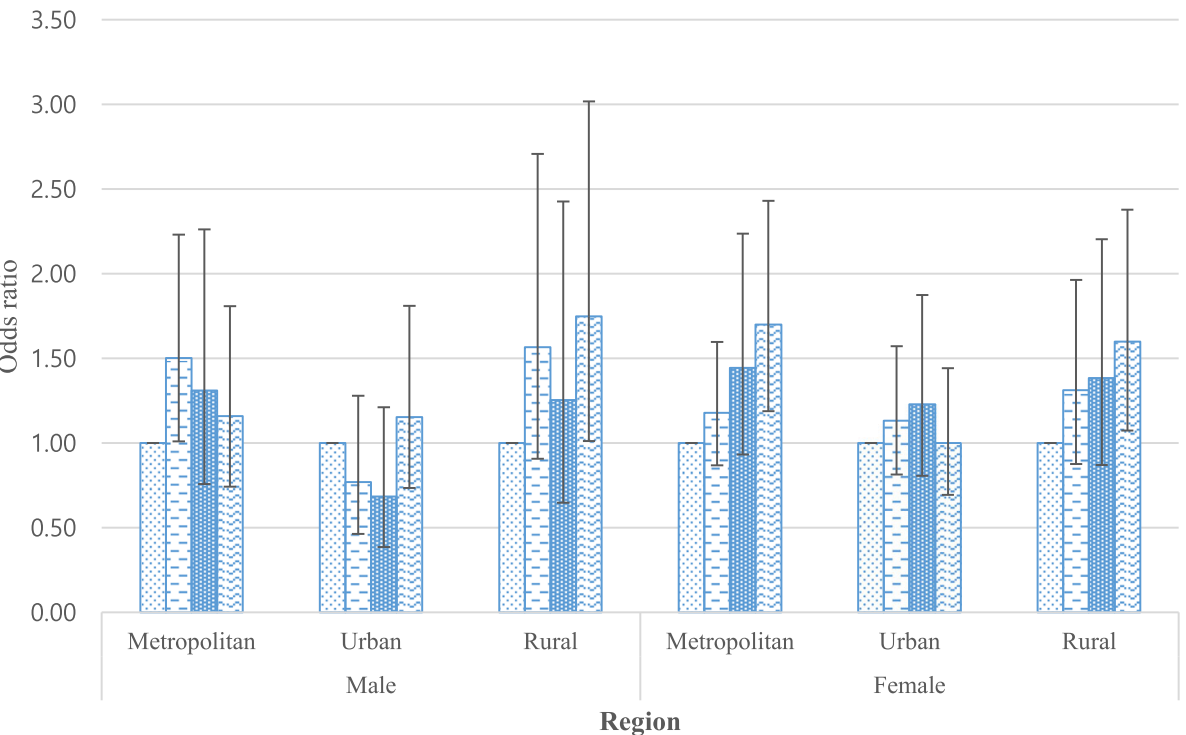

$$
\begin{array}{ll}
\square \text { Persistence of non-poverty }(\mathrm{NN}) & \square \text { Exiting from poverty (PN) } \\
\text { Transition to poverty (NP) } & \square \text { Persistence of poverty (PP) }
\end{array}
$$

Fig. 1 Results of subgroup analysis of poverty transitions to frailty stratified by region. Control variables include age, sex, marital status, number of household, educational level, current economic activity, health insurance type, household income, smoking, drinking, perceived health status, chronic disease, disability, ADL, IADL, cognitive impairment, frailty in the previous year. 95\% confidence interval

change, particularly income loss, is significantly associated with health [24]. There could be several reasons for poverty transition; however, sudden or unpredictable financial loss would be particularly damaging to health. For example, if poverty transitions occur because of unexpected job loss, it could cause not only financial loss but also acute disappointment, which could result in depression or social isolation [26]. It is not surprising that age is strongly associated with frailty. The present findings showed a graded association with increasing age, which is supported by previous studies [6, 27, 28]. The findings from the subgroup analysis demonstrated that middle-aged women did not show a significant association with frailty, suggesting that older women are more vulnerable to poverty transitions. As has been well studied in the literature $[29,30]$, socioeconomic variables including education and income levels, which are related to poverty, are also associated with frailty. For both men and women, being unmarried or living without spouse was associated with frailty. With older age, those who experienced bereavement or widowhood reported feeling more depressive symptoms and lower social ties, which may negatively affect their health [31]. Our findings were consistent with those of a previous study which found that being cognitively impaired increased the probability of frailty [32]. Furthermore, those who reported being dependent for their ADL and IADL had a higher probability of frailty $[18,33,34]$.
Although these findings elucidate how transitions in poverty status affect frailty, they should be interpreted with caution because of several limitations. First, the frailty scale used in the KLoSA was developed and validated only in Korea. However, it measures physical, psychological, and social determinants, thereby offering a broader approach to explaining frailty. Second, although our study was based on longitudinal data with repeated observations at the individual level over a period, we could not determine a perfect causal relationship between poverty transitions and frailty. Third, measurement errors due to recall bias might exist because of subjective and inaccurate responses by the respondents. Despite these limitations, our study has several strengths. The KLoSA is a South Korean panel study focusing on the elderly that has been verified by experts to have statistical validity and national representativeness. We measured poverty prevalence based on actual data calculated by Statistics Korea for greater reliability.

Based on the present findings, those who transition into poverty and stay persistently in poverty are at high risk of frailty, particularly women aged over 65 years. Previous studies have shown that better management and intervention may prevent the progress of frailty or increase the chances of recovering from frailty [35]. Providing care to frail individuals is difficult because of their vulnerability to deterioration, complex comorbidities, and increased social needs [36, 37]. Furthermore, frailty 
is influenced by multiple factors and their complex interactions, which accumulate over time. Thus, future research on the various aspects of frailty and how they are influenced by socioeconomic and cultural determinants would provide a better understanding of frailty in older age.

\section{Conclusions}

To our knowledge, this is one of the few studies investigating the effects of poverty transitions on frailty. Our study aims to expand the knowledge regarding frailty in socioeconomically vulnerable groups. The findings suggest that experiencing poverty transitions, entering poverty, and persistently being in poverty increase the risk of frailty. This study can contribute to the development of intervention strategies to better identify frail individuals who may be at greater risk of negative effects on health.

\section{Abbreviations}

ADL: Activities of daily living; GEE: Generalized estimating equation; IADL: Instrumental activities of daily living; OECD: Organization for Economic Co-operation and Development; KLoSA: Korean Longitudinal Study of Ageing

\section{Acknowledgments}

The authors would like to thank the participants and interviewers who contributed to conducting the Korean Longitudinal Study of Ageing. We would also like to thank Chang-O Kim and Sunwoo Duck who developed the frailty instrument employed in this research.

\section{Authors' contributions}

HMY, HJL, DWL, and ECP were involved in designing the study. HMY, HJL, and DWL contributed to the analysis and manuscript. ECP reviewed the study overall and performed significant editing of the manuscript. All authors have read and approved the final manuscript.

\section{Funding}

Not applicable.

\section{Availability of data and materials}

The data used in the study are available at https://survey.keis.or.kr/eng/klosa/ databoard/List.jsp

\section{Ethics approval and consent to participate}

The KLoSA study was approved by the National Statistical Office (Approval number: 33602) and was conducted after acquiring verbal consent of participants in the study. Because the KLOSA database has been released to the public for scientific use, ethical approval was not required for the study.

\section{Consent for publication}

There are no details of individual participants in the manuscript.

\section{Competing interests}

The authors declare that they have no competing interests.

\section{Author details}

1Department of Public Health, Yonsei University Graduate School, 50 Yonsei-ro, Seodaemun-gu, Seoul 03722, Republic of Korea. ${ }^{2}$ Institute of Health Services Research, Yonsei University, 50 Yonsei-ro, Seodaemun-gu, Seoul 03722, Republic of Korea. ${ }^{3}$ Department of Preventive Medicine and Institute of Health Services Research, Yonsei University College of Medicine, 50 Yonsei-ro, Seodaemun-gu, Seoul 03722, Republic of Korea.
Received: 2 January 2020 Accepted: 17 March 2020

Published online: 15 April 2020

\section{References}

1. United Nations. World population ageing 2019 highlights. 2019.

2. Statistics Korea. Prospective population estimation (2017-2067). 2019.

3. Fried L, Ferrucci L, Darer J, Williamson J, Anderson G. Untangling the concepts of disability, frailty, and comorbidity: implications for improved targeting and care. J Gerontol A Biol Sci Med Sci. 2004;59:M255-M63.

4. Clegg A, Young J, lliffe S, Rikkert M, Rockwood K. Frailty in elderly people. Lancet. 2013;381:752-62.

5. Fried L, Tangen C, Walston J, Newman A, Hirsch C, Gottdiener J, et al. Frailty in older adults: evidence for a phenotype. J Gerontol. 2001:56:M146-56.

6. Feng Z, Lugtenberg M, Franse C, Fang X, Hu S, Jin C, et al. Risk factors and protective factors associated with incident or increase of frailty among community-dwelling older adults: a systematic review of longitudinal studies. PLoS One. 2017;12:e0178383.

7. Hoogendijk $E$, van Hout $H$, Heymans $M$, van der Horst $H$, Frijters D, Broese van Groenou M, et al. Explaining the association between educational level and frailty in older adults: results from a 13-year longitudinal study in the Netherlands. Ann Epidemiol. 2014;24:538-44 e2.

8. Hoogendijk E, Rijnhart J, Kowal P, Perez-Zepeda M, Cesari M, Abizanda P, et al. Socioeconomic inequalities in frailty among older adults in six lowand middle-income countries: results from the WHO study on global AGEing and adult health (SAGE). Maturitas. 2018;115:56-63.

9. Watts $P$, Blane D, Netuveli. G.minimum income for healthy living and frailty in adults over 65 years old in the English Longitudinal Study of Ageing: a population-based cohort study. BMJ Open. 2019;9:e025334.

10. Stolz E, Mayerl H, Waxenegger A, Freidl W. Explaining the impact of poverty on old-age frailty in Europe: material, psychosocial and behavioural factors. Eur J Pub Health. 2017;27:1003-9.

11. Organization for Economic Cooperation and Development. Poverty rate (indicator); 2020. https://doi.org/10.1787/0fe1315d-en.

12. Korea Employment Information Service. User's guide for the 2018 Korean Longitudinal Study of Ageing (KLoSA) 2018.

13. Song $E$, Shin $Y$. The effect of catastrophic health expenditure on the transition to poverty and the persistence of poverty in South Korea. Prevent Med Public Health. 2010;43:423-35.

14. Kim C, Sunwoo D. A frailty instrument to predic disability, institutionalization, and mortality: findings from the living profiles of older people survey. J Korean Gerontological Society. 2015;35:451-74.

15. Kim KJ, Shin J, Choi J, Won CW. Discrepancies in the prevalence of known frailty scales: Korean frailty and aging cohort study. Ann Geriatric Med Res. 2018;22:137-44

16. Kang $Y, N a$ D, Hahn $S$, Kang $Y$, Han S. A validity study on the Korean minimental state examination (K-MMSE) in dementia patients. J Korean Neurol Assoc. 1997;15:300-8.

17. Ko Y, Choi K. Prevalence of frailty and associated factors in Korean older women: the KLoSA study. J Women Aging. 2017;29:15-25.

18. Lee I, Chiu Y, Lee C. Health-functional indicators for the prediction of elderly frailty. J Ageing Res Clin Pract. 2017;6:88-93.

19. Hsu H. Relationship between frailty and cognitive function among older adults in Taiwan. J Frailty Aging. 2014;3:153-7.

20. Lynch J, Kaplan G, Shema S. Cumulative impact of sustained economic hardship on physical, cognitive, psychological, and social functioning. N Engl J Med. 1997;337:1889-95.

21. Saito M, Kondo K, Kondo N, Abe A, Ojima T, Suzuki K, et al. Relative deprivation, poverty, and subjective health: JAGES cross-sectional study. PloS one. 2014;9:e111169 e.

22. Collard R, Boter $H$, Schoevers $R$, Oude VR. Prevalence of frailty in community-dwelling older persons: a systematic review. J Am Geriatr Soc. 2012;60:1487-92.

23. Santos-Eggimann B, Cuenoud P, Spagnoli J, Junod J. Prevalence of frailty in middle-aged and older community-dwelling Europeans living in 10 countries. J Gerontol A Biol Sci Med Sci. 2009;64:675-81.

24. Benzeval M, Judge K. Income and health: the time dimension. Soc Sci Med. 2001:52:1371-90.

25. Murray S. Poverty and health. CMAJ. 2006;174:923.

26. Prause J, Dooley D, Huh J. Income volatility and psychological depression. Am J Community Psychol. 2009:43:57-70. 
27. Rockwood K, Song X, Mitnitski A. Changes in relative fitness and frailty across the adult lifespan: evidence from the Canadian National Population Health Survey. Can Med Assoc J. 2011;183:E487-94.

28. Carneiro J, Cardoso R, Durães M, Guedes M, Santos F, Costa F, et al. Frailty in the elderly: prevalence and associated factors. J Revista Brasileira de Enfermagem. 2017:70:747-52.

29. Szanton S, Seplaki C, Thorpe R, Allen J, Fried L. Socioeconomic status is associated with frailty: the Women's health and aging studies. J Epidemiol Community Health. 2010;64:63-7.

30. Woods N, LaCroix A, Gray S, Aragaki A, Cochrane B, Brunner R, et al. Frailty: emergence and consequences in women aged 65 and older in the Women's Health Initiative observational study. J Am Geriatr Soc. 2005;53: 1321-30.

31. Stroebe M, Schut H, Stroebe W. Health outcomes of bereavement. Lancet. 2007;370:1960-73

32. Gale C, Cooper C, Sayer A. Prevalence of frailty and disability: findings from the English longitudinal study of ageing. Age Ageing. 2015:44:162-5.

33. de Carvalho Mello A, Engstrom E, Alves L. Health-related and sociodemographic factors associated with frailty in the elderly: a systematic literature review. Cad Saude Publica. 2014;30:1143-68.

34. Sousa A, Dias R, Maciel A, Guerra R. Frailty syndrome and associated factors in community-dwelling elderly in Northeast Brazil. Arch Gerontol Geriatr. 2012;54:e95-e101.

35. Lee J, Auyeung T, Leung J, Kwok T, Woo J. Transitions in frailty states among community-living older adults and their associated factors. J Am Med Dir Assoc. 2014;15:281-6.

36. Espinoza S, Walston J. Frailty in older adults: insights and interventions. Cleve Clin J Med. 2005;72:1105-12.

37. Fairhall N, Langron C, Sherrington C, Lord R, Kurrle S, Lockwood K, et al. Treating frailty-a practical guide. BMC Med. 2011;9:83.

\section{Publisher's Note}

Springer Nature remains neutral with regard to jurisdictional claims in published maps and institutional affiliations.

Ready to submit your research? Choose BMC and benefit from:

- fast, convenient online submission

- thorough peer review by experienced researchers in your field

- rapid publication on acceptance

- support for research data, including large and complex data types

- gold Open Access which fosters wider collaboration and increased citations

- maximum visibility for your research: over $100 \mathrm{M}$ website views per year

At $\mathrm{BMC}$, research is always in progress.

Learn more biomedcentral.com/submissions 